\title{
Measuring Closeness between Cayley Automatic Groups and Automatic Groups
}

\author{
Dmitry Berdinsky ${ }^{1,2}$ and Phongpitak Trakuldit ${ }^{1,2}$ \\ 1 Department of Mathematics, Faculty of Science, Mahidol University, Bangkok, \\ Thailand \\ 2 Centre of Excellence in Mathematics, Commission on Higher Education, Bangkok, \\ Thailand \\ berdinsky@gmail.com, p.trakuldit@gmail.com
}

\begin{abstract}
In this paper we introduce a way to estimate a level of closeness of Cayley automatic groups to the class of automatic groups using a certain numerical characteristic. We characterize Cayley automatic groups which are not automatic in terms of this numerical characteristic and then study it for the lamplighter group, the Baumslag-Solitar groups and the Heisenberg group.
\end{abstract}

Keywords: automatic groups, Cayley automatic groups, automatic structures, numerical characteristics of groups, lamplighter group, Heisenberg group, Baumslag-Solitar groups

\section{Introduction}

Cayley automatic groups had been introduced by Kharlampovich, Khoussainov and Miasnikov as a generalization of automatic groups [12. They are all finitely generated groups for which their directed labeled Cayley graphs are finite automata presentable structures (automatic structures) 13|14|15|16]; see, e.g., also 6]7|18|19|20|22 23]. In particular, Cayley automatic groups include all automatic groups in the sense of Thurston and others [10. Cayley automatic groups inherit the key algorithmic properties of automatic groups: the first order theory for a directed labeled Cayley graph of a Cayley automatic groups is decidable, the word problem in a Cayley automatic group is decidable in quadratic time 12 . The set of Cayley automatic groups comprise all finitely generated nilpotent groups of nilpotency class at most two [12, the Baumslag-Solitar groups 2, higher rank lamplighter groups 4 and all fundamental groups of 3-dimensional manifolds. This shows that Cayley automatic groups include important classes of groups.

In this paper we introduce the classes of Cayley automatic groups $\mathcal{B}_{f}$ defined by non-decreasing and non-negative functions $f$. Informally speaking, for any given group $G \in \mathcal{B}_{f}$, the function $f$ shows an upper bound for a level of closeness of the group $G$ to the class of automatic groups. In particular, if $f$ is identically equal to zero, then $G$ must be automatic. So, similarly to a growth function, one can consider $f$ as a numerical characteristic of the group $G$. Studying numerical 
characteristics of groups and relations between them is an important topic in group theory [24. In this paper we initiate study of this numerical characteristic. We first characterize non-automatic groups in terms of this characteristic. Then we study this characteristic for some non-automatic groups, namely, the lamplighter group $\mathbb{Z}_{2} \imath \mathbb{Z}$, the Baumslag-Solitar groups $B S(p, q)$, with $1 \leqslant p<q$, and the Heisenberg group $\mathcal{H}_{3}(\mathbb{Z})$. Another motivation to introduce this numerical characteristic is to address the problem of finding characterization for Cayley automatic groups by studying classes $\mathcal{B}_{f}$ for some functions $f$.

The paper is organized as follows. In Section 2 we recall the definitions of automatic and Cayley automatic groups. Then we give the definition of the classes of Cayley automatic groups $\mathcal{B}_{f}$ and show that it does not depend on the choice of generators. In Section 3 we give a characterization of non-automatic groups by showing that if $G \in \mathcal{B}_{f}$ is non-automatic, then $f$ must be unbounded. In Sections 4 and 5 we show that the Baumslag-Solitar groups $B S(p, q)$, with $1 \leqslant p<q$, and the lamplighter group $\mathbb{Z}_{2} \prec \mathbb{Z}$ are in the class $\mathcal{B}_{\mathfrak{i}}$, where $\mathfrak{i}$ is the identity function: $\mathfrak{i}(n)=n$. Moreover, we show that these groups cannot be elements of any class $\mathcal{B}_{f}$, if the function $f$ is less than $\mathfrak{i}$ in coarse sense (see Definition (4). In Section 6 we show that the Heisenberg group $\mathcal{H}_{3}(\mathbb{Z})$ is in the class $\mathcal{B}_{\mathfrak{e}}$, where $\mathfrak{e}$ is the exponential function: $\mathfrak{e}(n)=\exp (n)$. We then show that $\mathcal{H}_{3}(\mathbb{Z})$ cannot be an element of any class $\mathcal{B}_{f}$, if $f$ is less than the cubic root function $\sqrt[3]{n}$ in coarse sense. Section 7 concludes the paper.

\section{Preliminaries}

Let $G$ be a finitely generated infinite group. Let $A \subseteq G$ be a finite generating set of the group $G$. We denote by $S$ the set $S=A \cup A^{-1}$, where $A^{-1}$ is the set of the inverses of elements of $A$. For given elements $g_{1}, g_{1} \in G$, we denote by $d_{A}\left(g_{1}, g_{2}\right)$ the distance between the elements $g_{1}$ and $g_{2}$ in the Cayley graph $\Gamma(G, A)$. Similarly, we denote by $d_{A}(g)=d_{A}(e, g)$ the word length of $g$ with respect the generating set $A$. We denote by $\pi: S^{*} \rightarrow G$ the canonical mapping which sends every word $w \in S^{*}$ to the corresponding group element $\pi(w)=\bar{w} \in$ $G$.

We assume that the reader is familiar with the notion of finite automata and regular languages. For a given finite alphabet $\Sigma$ we put $\Sigma_{\diamond}=\Sigma \cup\{\diamond\}$, where $\diamond \notin \Sigma$ is a padding symbol. The convolution of $n$ words $w_{1}, \ldots, w_{n} \in \Sigma^{*}$ is the string $w_{1} \otimes \cdots \otimes w_{n}$ of length $\max \left\{\left|w_{1}\right|, \ldots,\left|w_{n}\right|\right\}$ over the alphabet $\Sigma_{\diamond}^{n}$ defined as follows. The $k$ th symbol of the string is $\left(\sigma_{1}, \ldots, \sigma_{n}\right)^{\top}$, where $\sigma_{i}, i=1, \ldots, n$ is the $k$ th symbol of $w_{i}$ if $k \leqslant\left|w_{i}\right|$ and $\diamond$ otherwise. The convolution $\otimes R$ of a $n$-ary relation $R \subseteq \Sigma^{* n}$ is defined as $\otimes R=\left\{w_{1} \otimes \cdots \otimes w_{n} \mid\left(w_{1}, \ldots, w_{n}\right) \in R\right\}$. We recall that a $n$-tape synchronous finite automaton is a finite automaton over the alphabet $\Sigma_{\diamond}^{n} \backslash\{(\diamond, \ldots, \diamond)\}$. We say that a $n$-ary relation $R \subseteq \Sigma^{* n}$ is regular if $\otimes R$ is accepted by a $n$-tape synchronous finite automaton.

Below we give a definition of automatic groups in the sense of Thurston and others [10]. 
Definition 1. We say that $G$ is automatic if there exists a regular language $L \subseteq S^{*}$ such that $\varphi=\left.\pi\right|_{L}: L \rightarrow G$ is a bijection and for every $a \in A$ the binary relation $R_{a}=\left\{\left(\varphi^{-1}(g), \varphi^{-1}(g a)\right) \mid g \in G\right\} \subseteq L \times L$ is regular.

Remark 2. In Definition 1 we required $\varphi$ to be bijective while in the original definition [10, Definition 2.3.1] $\varphi=\left.\pi\right|_{L}: L \rightarrow G$ is surjective but it is additionally required that the equality relation $R_{e}=\{(u, v) \in L \times L \mid \pi(u)=\pi(v)\}$ is regular. It can be seen that these two definitions are equivalent. Clearly, if a group $G$ is automatic in the sense of Definition 11 then it is automatic in the sense of 10. Definition 2.3.1]. Now, suppose that $G$ is automatic in the sense of [10, Definition 2.3.1]. Then there is a regular language $L \subseteq S^{*}$ for which the map $\varphi=\left.\pi\right|_{L}: L \rightarrow G$ is surjective and the relations $R_{a}, a \in A$ and $R_{e}$ are regular. Let $L^{\prime}=\left\{w \in L \mid\left(\forall u<_{\text {llex }} w\right) \pi(u) \neq \pi(w)\right\}$, where llex is a length-lexicographical order. Then $\varphi^{\prime}=\left.\pi\right|_{L^{\prime}}: L^{\prime} \rightarrow G$ is bijective and for every $a \in A$ the binary relation $R_{a}^{\prime}=\left\{\left(\varphi^{\prime-1}(g), \varphi^{\prime-1}(g a)\right) \mid g \in G\right\} \subseteq L^{\prime} \times L^{\prime}$ is regular. That is, $G$ is automatic in the sense of Definition 1

Definition 3. We say that $G$ is Cayley automatic if there exist a regular language $L \subseteq S^{*}$ and a bijection $\psi: L \rightarrow G$ such that for every $a \in A$ the binary relation $R_{a}=\left\{\left(\psi^{-1}(g), \psi^{-1}(g a)\right) \mid g \in G\right\} \subseteq L \times L$ is regular. We call $\psi: L \rightarrow G$ a Cayley automatic representation of $G$.

We denote by $\mathcal{A}$ and $\mathcal{C}$ the classes of all automatic and Cayley automatic groups, respectively. Clearly, $\mathcal{A} \subseteq \mathcal{C}$. However, $\mathcal{A}$ is a proper subset of $\mathcal{C}$ : for example, the lamplighter group, the Baumslag-Solitar groups and the Heisenberg group $\mathcal{H}_{3}(\mathbb{Z})$ are Cayley automatic, but not automatic. We will refer to $\mathbb{N}$ as the set of all positive integers. We denote by $\mathbb{R}^{+}$the set of all non-negative real numbers. Let $\mathfrak{F}$ be the following set of non-decreasing functions:

$\mathfrak{F}=\left\{f:[Q,+\infty) \rightarrow \mathbb{R}^{+} \mid[Q,+\infty) \subseteq \mathbb{N} \wedge \forall n(n \in \operatorname{dom} f \Longrightarrow f(n) \leqslant f(n+1))\right\}$.

Definition 4. Let $f, h \in \mathfrak{F}$. We say that $h \preceq f$ if there exist positive integers $K, M$ and $N$ such that $[N,+\infty) \subseteq \operatorname{dom} h \cap \operatorname{dom} f$ and $h(n) \leqslant K f(M n)$ for every integer $n \geqslant N$. We say that $h \asymp f$ if $h \preceq f$ and $f \preceq h$. We say that $h \prec f$ if $h \preceq f$ and $h \nsucc f$.

Let $G \in \mathcal{C}$ be a Cayley automatic group and $f \in \mathfrak{F}$. Let us choose some finite generating set $A \subseteq G$. For a given language $L \subseteq S^{*}$ and $n \in \mathbb{N}$ we denote by $L^{\leqslant n}$ the set of all words of length less than or equal to $n$ from the language $L$, i.e., $L^{\leqslant n}=\{w \in L|| w \mid \leqslant n\}$.

Definition 5. We say that $G \in \mathcal{B}_{f}$ if there exist a regular language $L \subseteq S^{*}$ and a Cayley automatic representation $\psi: L \rightarrow G$ such that for the function $h \in \mathfrak{F}$, defined by the equation

$$
h(n)=\max \left\{d_{A}(\pi(w), \psi(w)) \mid w \in L^{\leqslant n}\right\},
$$

the inequality $h \preceq f$ holds. 
We denote by $\mathcal{B}_{f}$ the class of all Cayley automatic groups $G$ for which $G \in \mathcal{B}_{f}$. Proposition 6 below shows that Definition 5 does not depend on the choice of generating set $A$.

Proposition 6. Definition 5 does not depend on the choice of generating set.

Proof. Let $A^{\prime} \subseteq G$ be another generating set of $G \in \mathcal{B}_{f}$. We put $S^{\prime}=A^{\prime} \cup A^{\prime-1}$. In order to simplify an exposition of our proof, we will assume that $e \in A^{\prime}$. Let us represent every element $g \in S$ by a word $w_{g} \in S^{\prime *}$, i.e., $\pi\left(w_{g}\right)=g$, for which the lengths of the words $\left|w_{g}\right|$ are the same for all $g \in S$. In order to make the lengths $w_{g}, g \in S$ equal, one can use $e \in S^{\prime}$ as a padding symbol. Let us canonically extend the mapping $g \mapsto w_{g}, g \in S$ to the monoid homomorphism $\xi: S^{*} \rightarrow S^{\prime *}$.

We remark that the definition of $\xi$ ensures that $\pi(\xi(w))=\pi(w)$ for $w \in S^{*}$. For a given Cayley automatic representation $\psi: L \rightarrow G$ for which $h \preceq f$, we construct a new Cayley automatic representation $\psi^{\prime}: L^{\prime} \rightarrow G$ as follows. We put $L^{\prime}=\xi(L) \subseteq S^{\prime *}$ and define a bijection $\psi^{\prime}: L^{\prime} \rightarrow G$ as $\psi^{\prime}=\psi \circ \tau$, where $\tau=\left(\left.\xi\right|_{L}\right)^{-1}$. It can be seen that $\psi^{\prime}$ is a Cayley automatic representation of $G$. Furthermore, for the function $h^{\prime} \in \mathfrak{F}$ defined by (1) with respect to $\psi^{\prime}$ we obtain that $h^{\prime} \preceq h$ which implies that $h^{\prime} \preceq f$. This proof can be generalized for the case when $e \notin A^{\prime}$.

We denote by $\mathbf{z} \in \mathfrak{F}$ the zero function: $\mathbf{z}(n)=0$ for all $n \in \mathbb{N}$. By Definition 5 we have that $\mathcal{B}_{\mathbf{z}}=\mathcal{A}$. Proposition 7 below shows some elementary properties of the classes $\mathcal{B}_{f}$.

Proposition 7. If $f \preceq g$, then $\mathcal{A} \subseteq \mathcal{B}_{f} \subseteq \mathcal{B}_{g} \subseteq \mathcal{C}$. If $f \asymp g$, then $\mathcal{B}_{f}=\mathcal{B}_{g}$.

Proof. By definition, every group of the class $\mathcal{B}_{g}$ is Cayley automatic, i.e., $\mathcal{B}_{g} \subseteq \mathcal{C}$. The inclusion $\mathcal{A} \subseteq \mathcal{B}_{f}$ follows from the fact that $\mathbf{z} \preceq f$ for every $f \in \mathfrak{F}$. The transitivity of the relation $\preceq$ on $\mathfrak{F}$ implies that if $f \preceq g$, then $\mathcal{B}_{f} \subseteq \mathcal{B}_{g}$. The fact that $f \asymp g$ implies $\mathcal{B}_{f}=\mathcal{B}_{g}$ is straightforward.

\section{Characterizing Non-Automatic Groups}

Let $G$ be a Cayley automatic group, $A \subseteq G$ be a finite generating set and $S=A \cup A^{-1}$. Given a word $w \in S^{*}$, for a non-negative integer $t$ we put $w(t)$ to be the prefix of $w$ of a length $t$, if $t \leqslant|w|$, and $w(t)=w$, if $t>|w|$. Following the notation from [10], we denote by $\widehat{w}:[0, \infty) \rightarrow \Gamma(G, A)$ the corresponding path in the Cayley graph $\Gamma(G, A)$ defined as follows. If $t \geqslant 0$ is an integer, then $\widehat{w}(t)=\pi(w(t))$, and $\widehat{w}$ is extended to non-integer values of $t$ by moving along the respective edges with unit speed. Given words $w_{1}, w_{2} \in S^{*}$ and a constant $C_{0} \geqslant 0$, we say that the paths $\widehat{w_{1}}$ and $\widehat{w_{2}}$ are a uniform distance less than or equal to $C_{0}$ apart if $d_{A}\left(\widehat{w_{1}}(t), \widehat{w_{2}}(t)\right) \leqslant C_{0}$ for all non-negative integers $t$.

Theorem 8 below is a simplified modification of the theorem characterizing automatic groups due to Epstein et al. [10, Theorem 2.3.5]. This theorem follows from the existence of standard automata [10, Definition 2.3.3] for all elements of 
$A$. For the existence of standard automata it is enough to assume the solvability of the word problem in $G$. We recall that for Cayley automatic the word problem in $G$ is decidable [12, Theorem 8.1].

Theorem 8. (【10, Theorem 2.3.5]) Let $L \subseteq S^{*}$ be a regular language such that $\pi: L \rightarrow G$ is surjective. Assume that there is a constant $C_{0}$ such that for every $w_{1}, w_{2} \in L$ and $a \in A$ for which $\pi\left(w_{1}\right) a=\pi\left(w_{2}\right)$, the paths $\widehat{w_{1}}$ and $\widehat{w_{2}}$ are $a$ uniform distance less than or equal to $C_{0}$ apart. Then $G$ is an automatic group.

Let $d \in \mathfrak{F}$ be any bounded function which is not identically equal to the zero function $\mathbf{z}$. Although $\mathbf{z} \prec d$, the theorem below shows that the class $\mathcal{B}_{d}$ does not contain any non-automatic group.

Theorem 9. The class $\mathcal{B}_{d}=\mathcal{A}$. In particular, if for any function $f \in \mathfrak{F}$ the class $\mathcal{B}_{f}$ contains a non-automatic group, then $f$ must be unbounded.

Proof. Let us show that $\mathcal{B}_{d}=\mathcal{A}$. By Proposition [7, we only need to show that $\mathcal{B}_{d} \subseteq \mathcal{A}$. Assume that $G \in \mathcal{B}_{d}$. By Definition [5, there exists a Cayley automatic representation $\psi_{0}: L_{0} \rightarrow G$ for some $L_{0} \subseteq S^{*}$ such that, for the function $h_{0}(n)=$ $\max \left\{d_{A}\left(\pi(w), \psi_{0}(w)\right) \mid w \in L_{0}^{\leqslant n}\right\}, h_{0} \preceq d$. This implies that $d_{A}\left(\psi_{0}(w), \pi(w)\right)$ is bounded from above by some constant $K_{0}$ for all $w \in L_{0}$.

We put $L_{1}=S^{* \leqslant K_{0}}$. Let $L=L_{0} L_{1}$ be the concatenation of $L_{0}$ and $L_{1}$. The language $L$ is regular. For any given $g \in G, d_{A}\left(\pi\left(\psi_{0}^{-1}(g)\right), g\right) \leqslant K_{0}$. This implies that there is a word $u \in L_{1}$ such that, for the concatenation $w=\psi_{0}^{-1}(g) u$, $\pi(w)=g$. Therefore, the map $\pi: L \rightarrow G$ is surjective.

Let $w_{1}, w_{2} \in L$ be some words for which $\pi\left(w_{1}\right) a=\pi\left(w_{2}\right), a \in A$. There exist words $v_{1}, v_{2} \in L_{0}$ and $u_{1}, u_{2} \in L_{1}$ for which $w_{1}=v_{1} u_{1}$ and $w_{2}=v_{2} u_{2}$. We obtain that $d_{A}\left(\psi_{0}\left(v_{1}\right), \psi_{0}\left(v_{2}\right)\right) \leqslant d_{A}\left(\pi_{0}\left(v_{1}\right), \pi_{0}\left(v_{2}\right)\right)+2 K_{0} \leqslant d_{A}\left(\pi\left(w_{1}\right), \pi\left(w_{2}\right)\right)+$ $2 K_{0}+2 K_{0} \leqslant 4 K_{0}+1$. That is, there exists $g \in G$, for which $d_{A}(g) \leqslant 4 K_{0}+1$, such that $\psi_{0}\left(v_{1}\right) g=\psi_{0}\left(v_{2}\right)$. The pair $\left(v_{1}, v_{2}\right)$ is accepted by some two-tape synchronous automaton $M_{g}$.

Let $N_{g}$ be the number of states of $M_{g}$. Given a non-negative integer $t$, there exist words $p_{1}, p_{2} \in S^{*}$, for which the lengths $\left|p_{1}\right|,\left|p_{2}\right|$ are bounded from above by $N_{g}$, such that the pair $\left(v_{1}(t) p_{1}, v_{2}(t) p_{2}\right)$ is accepted by $M_{g}$; in particular, $v_{1}(t) p_{1}, v_{2}(t) p_{2} \in L_{0}$. We obtain that:

$$
\begin{array}{r}
d_{A}\left(\pi\left(v_{1}(t)\right), \pi\left(v_{2}(t)\right)\right) \leqslant d_{A}\left(\pi\left(v_{1}(t) p_{1}\right), \pi\left(v_{2}(t) p_{2}\right)\right)+\left|p_{1}\right|+\left|p_{2}\right| \leqslant \\
d_{A}\left(\psi_{0}\left(v_{1}(t) p_{1}\right), \psi_{0}\left(v_{2}(t) p_{2}\right)\right)+2 K_{0}+2 N_{g} \leqslant \\
d_{A}(g)+2 K_{0}+2 N_{g} \leqslant 6 K_{0}+2 N_{g}+1 .
\end{array}
$$

Therefore,

$$
\begin{array}{r}
d_{A}\left(\widehat{w_{1}}(t), \widehat{w_{2}}(t)\right)=d_{A}\left(\pi\left(w_{1}(t)\right), \pi\left(w_{2}(t)\right)\right) \leqslant d_{A}\left(\pi\left(v_{1}(t)\right), \pi\left(v_{2}(t)\right)\right)+2 K_{0} \leqslant \\
8 K_{0}+2 N_{g}+1 .
\end{array}
$$

There are only finitely many $g$ for which $d_{A}(g) \leqslant 4 K_{0}+1$, so $N_{g}$ can be bound by some constant $N_{0}$. Thus, for $C_{0}=8 K_{0}+2 N_{0}+1$, we obtain that $d_{A}\left(\widehat{w_{1}}(t), \widehat{w_{2}}(t)\right) \leqslant C_{0}$, that is, the paths $\widehat{w_{1}}$ and $\widehat{w_{2}}$ are a uniform distance $C_{0}$ apart. By Theorem 8, the group $G$ is automatic. The second statement of the theorem is straightforward. 


\section{The Baumslag-Solitar Groups}

Let us consider the Baumslag-Solitar groups $B S(p, q)=\left\langle a, t \mid t a^{p} t^{-1}=a^{q}\right\rangle$ with $1 \leqslant p<q$. These groups are not automatic, see Epstein et al. [10, Section 7.4], but they are Cayley automatic [2, Theorem 3]. The Cayley automatic representations of the Baumslag-Solitar groups constructed in [2, Theorem 3] use the normal form obtained from representing these groups as the HNN extensions 2 Corollary 2]. This normal form is shown in the following proposition.

Proposition 10. Any element $g \in B S(p, q)$ for $1 \leqslant p<q$ can be written uniquely as $g=\widetilde{w}(a, t) a^{k}$, where

$$
\widetilde{w}(a, t) \in\left\{t, a t, \ldots, a^{q-1} t, t^{-1}, a t^{-1}, \ldots, a^{p-1} t^{-1}\right\}^{*}
$$

is freely reduced and $k \in \mathbb{Z}$.

Let us now describe a modification of the Cayley automatic representation of $B S(p, q)$ constructed in [2, Theorem 3.2] which is compatible with Definition 3. We put $a_{1}=a, \ldots, a_{q-1}=a^{q-1}$. Let $A=\left\{a_{0}, a_{1}, \ldots, a_{q-1}, t\right\}$ and $S=A \cup A^{-1}=\left\{e, a_{1}, a_{2}, \ldots, a_{q-1}, a_{1}^{-1}, \ldots, a_{q-1}^{-1}, t, t^{-1}\right\}$. Given an element $g=\widetilde{w}(a, t) a^{k} \in B S(p, q)$, we construct the word $w=u v$ which is the concatenation of two words $u, v \in S^{*}$ defined as follows.

The word $u \in\left\{t, t^{-1}, a_{1}, \ldots, a_{q-1}\right\}^{*}$ is obtained from the corresponding word $\widetilde{w}(a, t)$ by changing the subwords $a t^{\epsilon}, \ldots, a^{q-1} t^{\epsilon}$ to the subwords $a_{1} t^{\epsilon}, \ldots, a_{q-1} t^{\epsilon}$, respectively, where $\epsilon=+1$ or $\epsilon=-1$. The word $v$ is obtained from the $q$-ary representation of $|k|$ by changing the 0 to $e$ and $1, \ldots, q-1$ to $a_{1}, \ldots, a_{q-1}$ and $a_{1}^{-1}, \ldots, a_{q-1}^{-1}$, if $k \geqslant 0$ and $k<0$, respectively. The set of all such words $w$ is a regular language $L \subseteq S^{*}$. Thus, we have constructed a bijection $\psi: L \rightarrow$ $B S(p, q)$.

By [2, Theorem 3.2], $\psi$ provides a Cayley automatic representation of $B S(p, q)$. It is worth noting that if $g \in B S(p, q)$ is an element for which $k=0$, then for $w=\psi^{-1}(g)$ we obtain that $\psi(w)=\pi(w)$. Let $\widetilde{A}=\{a, t\}$. We have the following metric estimates for the groups $B S(p, q)$.

Theorem 11. ([8, Theorem 3.2]) There exist constants $C_{1}, C_{2}, D_{1}, D_{2}>0$ such that for every element $g \in B S(p, q)$ for $1 \leqslant p<q$ written as $\widetilde{w}(a, t) a^{k}$, we have: $C_{1}(|\widetilde{w}|+\log (|k|+1))-D_{1} \leqslant d_{\widetilde{A}}(g) \leqslant C_{2}(|\widetilde{w}|+\log (|k|+1))+D_{2}$.

Remark 12. The normal form for the elements of $B S(p, q)$ used in 8 is almost the same as in Proposition 10 modulo the choice for the range of powers of $a$. Namely, any element $g \in B S(p, q)$ for $1 \leqslant p<q$ can also be written uniquely as $g=w(a, t) a^{k}$ such that

$$
\begin{array}{r}
w(a, t) \in\left\{t, a t, a^{2} t, \ldots, a^{\alpha} t, a^{-1} t, a^{-2} t, \ldots, a^{-\beta} t, t^{-1}, a t^{-1}, a^{2} t^{-1}, \ldots,\right. \\
\left.a^{\gamma} t^{-1}, t^{-1}, a^{-1} t^{-1}, a^{-2} t^{-1}, \ldots, a^{-\delta} t^{-1}\right\}^{*}
\end{array}
$$

is freely reduced, where $\alpha=\left\lfloor\frac{q}{2}\right\rfloor, \beta=\left\lfloor\frac{q-1}{2}\right\rfloor, \gamma=\left\lfloor\frac{p}{2}\right\rfloor$ and $\delta=\left\lfloor\frac{p-1}{2}\right\rfloor$, see [8. Lemma 3.1]. It can be seen that the metric estimates obtained in [8] for the normal form $w(a, t) a^{k}$ remain valid for the normal form $\widetilde{w}(a, t) a^{k}$ modulo changing the constants $C_{1}$ and $C_{2}$. 
It follows from Theorem 11 that there exist constants $C_{1}^{\prime}, C_{2}^{\prime}, D_{1}^{\prime}, D_{2}^{\prime}>0$ such that for every element $g \in B S(p, q)$ and for the corresponding word $\psi^{-1}(g)=u v$ we have

$$
C_{1}^{\prime}(|u|+|v|)-D_{1}^{\prime} \leqslant d_{A}(g) \leqslant C_{2}^{\prime}(|u|+|v|)+D_{2}^{\prime} .
$$

Theorem 13. Given $p$ and $q$ for which $1 \leqslant p<q$, the Baumslag-Solitar group $B S(p, q) \in \mathcal{B}_{\mathfrak{i}}$. Moreover, for any $f \prec \mathfrak{i}, B S(p, q) \notin \mathcal{B}_{f}$.

Proof. For given $p$ and $q$ for which $1 \leqslant p<q$ let us consider the Cayley automatic representation $\psi: L \rightarrow B S(p, q)$ constructed above. Let $h$ be the function given by (1) with respect to this Cayley automatic representation. We will show that $h \preceq \mathfrak{i}$ (in fact one can verify that $h \asymp \mathfrak{i}$ ). Let $w=u v \in L^{\leqslant n}$ and $g=\psi(w)$ be the corresponding group element of $B S(p, q)$. By (2), there exists a constant $C$ such that $d_{A}(g) \leqslant C(|u|+|v|)=C|w|$. Therefore, $d_{A}(\pi(w), \psi(w)) \leqslant n+d_{A}(g) \leqslant$ $(C+1) n$. Therefore, $h \preceq \mathfrak{i}$ which implies that $B S(p, q) \in \mathcal{B}_{\mathfrak{i}}$.

Let us show now the second statement of the theorem. Suppose that $B S(p, q) \in$ $\mathcal{B}_{f}$ for some $f \prec \mathfrak{i}$. Then there exists a Cayley automatic representation $\psi^{\prime}$ : $L^{\prime} \rightarrow B S(p, q)$ for which $h^{\prime} \preceq f$, where $h^{\prime}$ is given by (1). We have $h^{\prime} \prec \mathfrak{i}$. We recall that for a group $\langle X \mid R\rangle$ given by a set of generators $X$ and a set of relators $R$ the Dehn function is given by $D(n)=\max _{u \in U_{n}}\{\operatorname{area}(u)\}$, where $U_{n}=\left\{u \in\left(X \cup X^{-1}\right)^{*}|\pi(u)=e \wedge| u \mid \leqslant n\right\}$ is the set of words of the length at most $n$ representing the identity of the group $\langle X \mid R\rangle$ and area $(u)$ is the combinatorial area of $u$ which is the minimal $k$ for which $u=\prod_{i=1}^{k} v_{i} r_{i}^{ \pm 1} v_{i}^{-1}$ in the free group $F(X)$, where $r_{i} \in R$.

Let $w \in\left\{a, a^{-1}, t, t^{-1}\right\}^{*}$ be a word representing the identity in $B S(p, q)$ for which $|w| \leqslant n$. The word $w$ corresponds to a loop in the Cayley graph $B S(p, q)$ with respect to the generators $a, t$. Similarly to the argument in the proof of 10 Theorem 2.3.12], it can be seen that the loop $w$ can be subdivided into at most $K_{0} n^{2}$ loops of length at most $\ell(n)=4 h^{\prime}\left(K_{0} n\right)+K_{1}$ for some integer constants $K_{0}$ and $K_{1}$. Therefore, $D(n) \leqslant K_{0} n^{2} D(\ell(n))$ which implies that $D(n) \preceq n^{2} D(\ell(n))$.

For the group $B S(p, q)$ the Dehn function is at most exponential (see 10 , $\S 7.4])$, i.e., $D(n) \leqslant \lambda^{n}$ for some constant $\lambda$. Therefore, $D(n) \preceq n^{2} \lambda^{\ell(n)}$. Clearly, $\ell \preceq h^{\prime}$ which implies that $\ell \prec \mathfrak{i}$. Let us show that $n^{2} \lambda^{\ell(n)} \prec \mathfrak{e}$. It can be seen that $n^{2} \lambda^{\ell(n)} \preceq \mathfrak{e}$. Assume that $\mathfrak{e} \preceq n^{2} \lambda^{\ell(n)}$. Then, for all sufficiently large $n$ and some constants $K$ and $M$ we have: $\exp (n) \leqslant K n^{2} \lambda^{\ell(M n)}$. This implies that $n-2 \ln n-\ln K \leqslant(\ln \lambda) \ell(M n)$. Clearly, $\frac{n}{2} \leqslant n-2 \ln n-\ln K$ for all sufficiently large $n$, and, therefore, $n \leqslant(2 \ln \lambda) \ell(M n)$. This implies that $\mathfrak{i} \preceq \ell$ which contradicts to the inequality $\ell \prec \mathfrak{i}$. Thus, $D(n) \preceq n^{2} \lambda^{\ell(n)} \prec \mathfrak{e}$ which implies that $D(n) \prec \mathfrak{e}$. The last inequality contradicts to the fact that for the group $B S(p, q)$ the Dehn function is at least exponential, i.e., $D(n) \geqslant \mu^{n}$ for some constant $\mu$ (see [10, § 7.4]) which implies that $\mathfrak{e} \preceq D(n)$.

\section{The Lamplighter Group}

The lamplighter group is the wreath product $\mathbb{Z}_{2} \imath \mathbb{Z}$ of the cyclic group $\mathbb{Z}_{2}$ and the infinite cyclic group $\mathbb{Z}$. For the definition of the wreath product of groups 
we refer the reader to [11. Let $t$ be a generator of the cyclic group $\mathbb{Z}=\langle t\rangle$ and $a$ be the nontirival element of the group $\mathbb{Z}_{2}$. The canonical embeddings of the groups $\mathbb{Z}_{2}$ and $\mathbb{Z}$ into the wreath product $\mathbb{Z}_{2} \nmid \mathbb{Z}$ enable us to consider $\mathbb{Z}_{2}$ and $\mathbb{Z}$ as the subgroups of $\mathbb{Z}_{2} \imath \mathbb{Z}$. With respect to the generators $a$ and $t$, the lamplighter group has the presentation $\left\langle a, t \mid\left[t^{i} a t^{-i}, t^{j} a t^{-j}\right], a^{2}\right\rangle$. The lamplighter group is not finitely presented [1, and, therefore, it is not automatic due to [10, Theorem 2.3.12].

The elements of the lamplighter group have the following geometric interpretation. Every element of the lamplighter group corresponds to a bi-infinite string of lamps, indexed by integers $i \in \mathbb{Z}$, each of which is either lit or unlit, such that only finite number of lamps are lit, and the lamplighter pointing at the current lamp $i=m$. The identity of the lamplighter group corresponds to the configuration when all lamps are unlit and the lamplighter points at the lamp positioned at the origin $m=0$.

The right multiplication by a changes the state of the current lamp. The right multiplication by $t$ (or $t^{-1}$ ) moves the lamplighter to the right $m \mapsto m+1$ (or to the left $m \mapsto m-1$ ). The elements of the subgroup $\mathbb{Z} \leqslant \mathbb{Z}_{2} \imath \mathbb{Z}$ are the configurations for which all lamps are unlit. For the elements of the subgroup $\mathbb{Z}_{2} \leqslant \mathbb{Z}_{2} \succ \mathbb{Z}$ all lamps, apart from the one at the origin, are unlit and the lamplighter points at the lamp positioned at the origin, which can be either lit or unlit.

For any given integer $i \in \mathbb{Z}$ we put $a_{i}=t^{i} a t^{-i}$. The group element $a_{i}$ corresponds to the configuration when the lamp at the position $i$ is lit, all other lamps are unlit and the lamplighter points at the origin $m=0$. Let $g$ be an element of the lamplighter group. The 'right-first' and the 'left-first' normal forms of $g$ are defined as follows:

$$
\begin{aligned}
& r f(g)=a_{i_{1}} a_{i_{2}} \ldots a_{i_{k}} a_{-j_{1}} a_{-j_{2}} \ldots a_{-j_{l}} t^{m}, \\
& l f(g)=a_{-j_{1}} a_{-j_{2}} \ldots a_{-j_{l}} a_{i_{1}} a_{i_{2}} \ldots a_{i_{k}} t^{m},
\end{aligned}
$$

where $i_{k}>\cdots>i_{2}>i_{1} \geqslant 0, j_{l}>\cdots>j_{1}>0$ and the lamplighter points at the position $m$ (see [9]). For the element $g$ the lit lamps are at the positions $-j_{l}, \ldots,-j_{1}, i_{1}, \ldots, i_{k}$ and the lamplighter points at the position $m$. In 'rightfirst' normal form the lamplighter moves to the right illuminating the appropriate lamps until it reaches the lamp at the position $i_{k}$. Then it moves back to the origin, and then further to the left illuminating the appropriate lamps until it reaches the lamp at the position $-j_{l}$. After that the lamplighter moves to the position $m$. Let $A=\{a, t\}$ and $S=\left\{a, a^{-1}, t, t^{-1}\right\}$.

Proposition 14. ([9, Proposition 3.2]) The word length of the element $g$ with respect to the generating set $A$ is given by

$$
d_{A}(g)=k+l+\min \left\{2 i_{k}+j_{l}+\left|m+j_{l}\right|, 2 j_{l}+i_{k}+\left|m-i_{k}\right|\right\} .
$$

Some Cayley automatic representations of $\mathbb{Z}_{2} \prec \mathbb{Z}$ had been obtained in $12 / 213$. Let us now construct a new Cayley automatic representation of $\mathbb{Z}_{2} \prec \mathbb{Z}$ using the 'right-first' normal form which is compatible with Definition 3. For a given 
element $g$ of the lamplighter group we construct the word $w=u^{\prime} v^{\prime}$ which is the concatenation of two words $u^{\prime}, v^{\prime} \in S^{*}$. The words $u^{\prime}$ and $v^{\prime}$ are obtained from the words $u$ and $v$, defined below, by canceling adjacent opposite powers of $t$. Assume first that $m \geqslant 0$.

- Suppose that $\left\{i_{1}, \ldots, i_{k}\right\}=\varnothing$ or $\left\{i_{1}, \ldots, i_{k}\right\} \neq \varnothing$ and $m>i_{k}$. We put $u=t^{i_{1}} a t^{-i_{1}} \ldots t^{i_{k}} a t^{-i_{k}} t^{m} a a$. We put $v=t^{-j_{1}} a t^{j_{1}} \ldots t^{-j_{l}} a$.

- Suppose that $\left\{i_{1}, \ldots, i_{k}\right\} \neq \varnothing$ and $m \leqslant i_{k}$. If $m=i_{n}$ for some $n=1, \ldots, k$, then we put $u=t^{i_{1}} a t^{-i_{1}} \ldots t^{i_{n}} a a a t^{-i_{n}} \ldots t^{i_{k}} a$. Otherwise, either $m<i_{1}$ or there exists $q=1, \ldots, k-1$ for which $i_{q}<m<i_{q+1}$. In the first case we put $u=t^{m} a a t^{-m} t^{i_{1}} a t^{-i_{1}} \ldots t^{i_{k}} a$. In the latter case we put $u=$ $t^{i_{1}} a t^{-i_{1}} \ldots t^{i_{q}} a t^{-i_{q}} t^{m} a a t^{-m} t^{i_{q+1}} a t^{-i_{q+1}} \ldots t^{i_{k}} a$. The word $v$ is the same as above.

Assume now that $m<0$.

- Suppose that $\left\{j_{1}, \ldots, j_{l}\right\}=\varnothing$ or $\left\{j_{1}, \ldots, j_{l}\right\} \neq \varnothing$ and $m<-j_{l}$. We put $v=t^{-j_{1}} a t^{j_{1}} \ldots t^{-j_{l}} a t^{j_{l}} t^{m} a a$. We put $u=t^{i_{1}} a t^{-i_{1}} \ldots t^{i_{k}} a$.

- Suppose that $\left\{j_{1}, \ldots, j_{l}\right\} \neq \varnothing$ and $m \geqslant-j_{l}$. If $m=-j_{n}$ for some $n=$ $1, \ldots, l$, then we put $v=t^{-j_{1}} a t^{j_{1}} \ldots t^{-j_{n}} a a a t^{j_{n}} \ldots t^{-j_{l}} a$. Otherwise, either $m>-j_{1}$ or there exists $q=1, \ldots, l-1$ for which $-j_{q}>m>-j_{q+1}$. In the first case we put $v=t^{m} a a t^{-m} t^{-j_{1}} a t^{j_{1}} \ldots t^{-j_{l}} a t^{j_{l}}$. In the latter case we put $v=t^{-j_{1}} a t^{j_{1}} \ldots t^{-j_{q}} a t^{j_{q}} t^{m} a a t^{-m^{2}} t^{-j_{q+1}} a t^{j_{q+1}} \ldots t^{-j_{l}} a$. The word $u$ is the same as above.

Let us show two simple examples. Suppose first that the lit lamps are at the positions $-1,0,2$ and the lamplighter is at the position $m=1$. Then, for the corresponding group element, the word $w$ is ataatat ${ }^{-1} a$. Suppose now that the lit lamps are at the positions $-1,1$ and the lamplighter is at the position $m=-1$. Then, for the corresponding group element, the word $w$ is $t a t^{-1} a a a$. The set of all such words $w$ forms some language $L \subseteq S^{*}$. Thus, we have constructed the bijection $\psi: L \rightarrow \mathbb{Z}_{2} \prec \mathbb{Z}$.

It can be verified that $L$ is a regular language and $\psi$ provides a Cayley automatic representation of the lamplighter group in the sense of Definition 3 We note that in the Cayley automatic representation $\psi: L \rightarrow \mathbb{Z}_{2} \prec \mathbb{Z}$ constructed above we use the subwords $a a$ and $a a a$ to specify the lamplighter position. We use $a a$ and $a a a$ if the lamp, the lamplighter is pointing at, is unlit and lit, respectively. It is worth noting that if $g \in \mathbb{Z}_{2} \succ \mathbb{Z}$ is an element for which all lamps at negative positions $j<0$ are unlit and $m \geqslant i_{k}$, then for $w=\psi^{-1}(g)$ we obtain that $\pi(w)=\psi(w)$. That is, on a certain infinite subset of $L$ the maps $\pi$ and $\psi$ coincide.

Theorem 15. The lamplighter group $\mathbb{Z}_{2} \prec \mathbb{Z} \in \mathcal{B}_{\mathfrak{i}}$. Moreover, for any $f \prec \mathfrak{i}$, $\mathbb{Z}_{2} \imath \mathbb{Z} \notin \mathcal{B}_{f}$

Proof. Let us consider the Cayley automatic representation $\psi: L \rightarrow \mathbb{Z}_{2}$ 乙 $\mathbb{Z}$ constructed above. Let $h$ be the function given by (1) with respect to the Cayley automatic representation $\psi$. We will show that $h \preceq \mathfrak{i}$ (in fact one can verify that 
$h \asymp \mathfrak{i})$. For a given $n$ let $w \in L^{\leqslant n}$ be a word and $g=\psi(w)$ be the corresponding group element of $\mathbb{Z}_{2} \nmid \mathbb{Z}$. Clearly, we have that $d_{A}(\pi(w), \psi(w)) \leqslant n+d_{A}(g)$. Therefore, it suffices to show that $d_{A}(g) \leqslant C n$ for some constant $C$.

It follows from the construction of $w=\psi^{-1}(g)$ that if $m \geqslant 0$, then $|w|=$ $k+l+\max \left\{m, i_{k}\right\}+j_{l}+2$, and if $m<0$, then $|w|=k+l+\max \left\{-m, j_{l}\right\}+i_{k}+2$. By Proposition 14, we obtain that $d_{A}(g) \leqslant 3|w| \leqslant 3 n$. Therefore, $h \preceq \mathfrak{i}$ which implies that $\mathbb{Z}_{2} \nmid \mathbb{Z} \in \mathcal{B}_{\mathfrak{i}}$. Let us show the second statement of the theorem. For a given $m>0$, let $R_{m}$ be the following set of relations $R_{m}=\left\{a^{2}\right\} \cup\left\{\left[t^{i} a t^{-i}, t^{j} a t^{-j}\right] \mid-\right.$ $m \leqslant i<j \leqslant m\}$. We first notice that for any loop $w \in S^{*},|w| \leqslant l$ in the lamplighter group $\mathbb{Z}_{2} \imath \mathbb{Z}$ the word $w$ can be represented as a product of conjugates of the relations from $R_{l}$, i.e., the identity $w=\prod_{i=1}^{k} v_{i} r_{i}^{ \pm 1} v_{i}^{-1}$ holds in the free group $F(A)$ for some $v_{i} \in S^{*}$ and $r_{i} \in R_{l}, i=1, \ldots, k$.

Suppose now that $\mathbb{Z}_{2} \imath \mathbb{Z} \in \mathcal{B}_{f}$ for some $f \prec i$. Similarly to Theorem 13 we obtain that then there exists a function $\ell \prec \mathfrak{i}$ such that any loop $w$ of the length less than or equal to $n$ can be subdivided into loops of the length at most $\ell(n)$. Therefore, for any loop given by a word $w \in S^{*},|w| \leqslant n$, the identity $w=\prod_{i=1}^{k} v_{i} r_{i}^{ \pm 1} v_{i}^{-1}$ holds in the free group $F(A)$ for some $v_{i} \in S^{*}$ and $r_{i} \in R_{\ell(n)}$, $i=1, \ldots, k$. In particular, every relation from $R_{n}$ can be expressed as a product of conjugates of the relations from $R_{\ell(8 n+4)}$ (the longest relation from $R_{n}$ is $\left[t^{-n} a t^{n}, t^{n} a t^{-n}\right]$ which has the length $\left.8 n+4\right)$. However, not every relation from $R_{n}$ can be expressed as a product of conjugates of the relations from $R_{n-1} \subset R_{n}$ because the groups $G_{n}=\left\langle a, t \mid R_{n}\right\rangle$ and $G_{n-1}=\left\langle a, t \mid R_{n-1}\right\rangle$ are not isomorphic. This implies the inequality $\ell(8 n+4) \geqslant n$ leading to a contradiction with $\ell \prec \mathfrak{i}$. The fact that $G_{n}=\left\langle a, t \mid R_{n}\right\rangle$ and $G_{n-1}=\left\langle a, t \mid R_{n-1}\right\rangle$ are not isomorphic can be shown as follows.

The group $G_{n}$ can be represented as $G_{n}=\left\langle a_{-n}, \ldots, a_{0}, \ldots, a_{n}\right| a_{0}^{2} ; a_{i-1}=$ $\left.t^{-1} a_{i} t, i=-(n-1), \ldots, n ;\left[a_{i}, a_{j}\right], i, j=-n, \ldots, n\right\rangle$, so $G_{n}$ is the HNN extension of the base group $\bigoplus_{i=-n}^{n} \mathbb{Z}_{2}=\left\langle a_{-n}, \ldots, a_{n} \mid a_{i}^{2},\left[a_{i}, a_{j}\right]\right\rangle$ relative to the isomorphism $\varphi_{n}$ between the subgroups $A_{n}, B_{n} \leqslant G_{n}$ generated by $a_{-(n-1)}, \ldots, a_{n}$ and $a_{-n}, \ldots, a_{n-1}$, respectively, for which $\varphi_{n}: a_{i} \mapsto a_{i-1}, i=-(n-1), \ldots, n$. As a consequence of Britton's lemma [17, we have the property that every finite subgroup of an HNN extension is conjugate to a finite subgroup of its base group. Assuming that $G_{n+1}$ and $G_{n}$ are isomorphic, we obtain that $\bigoplus_{i=-(n+1)}^{n+1} \mathbb{Z}_{2}$ can be embedded into $\bigoplus_{i=-n}^{n} \mathbb{Z}_{2}$ which leads to a contradiction.

\section{The Heisenberg Group}

The Heisenberg group $\mathcal{H}_{3}(\mathbb{Z})$ is the group of all matrices of the form:

$$
\left(\begin{array}{lll}
1 & x & z \\
0 & 1 & y \\
0 & 0 & 1
\end{array}\right),
$$

where $x, y$ and $z$ are integers. Every element $g \in \mathcal{H}_{3}(\mathbb{Z})$ corresponds to a triple $(x, y, z)$. Let $s$ be a group element of $\mathcal{H}_{3}$ corresponding to the triple $(1,0,0)$, 
$p$ corresponding to $(0,1,0)$, and $q$ corresponding to $(0,0,1)$. If $g$ corresponds to a triple $(x, y, z)$, then $g s, g p$ and $g q$ correspond to the triples $(x+1, y, z)$, $(x, y+1, x+z)$ and $(x, y, z+1)$, respectively. The observation that $\mathcal{H}_{3}$ is not an automatic group but its Cayley graph is automatic was first made by Sénizergues.

The Heisenberg group $\mathcal{H}_{3}$ is isomorphic to the group $\langle s, p, q| s^{-1} p^{-1} s p=$ $q, s q=q s, p q=q p\rangle$, and it can be generated by the elements $s$ and $p$. The exact distance formula on $\mathcal{H}_{3}(\mathbb{Z})$ for the generating set $\{s, p\}$ is obtained in [5. Theorem 2.2]. However, for our purposes it is enough to have the metric estimates which the reader can find in [21, Proposition 1.38]. Let $A=\{e, s, p, q\}$ and $S=A \cup A^{-1}=\left\{e, s, p, q, s^{-1}, p^{-1}, q^{-1}\right\}$.

Proposition 16. ([21, Proposition 1.38]) There exist constants $C_{1}$ and $C_{2}$ such that for an element $g \in \mathcal{H}_{3}$ corresponding to a triple $(x, y, z)$ we have

$$
C_{1}(|x|+|y|+\sqrt{|z|}) \leqslant d_{A}(g) \leqslant C_{2}(|x|+|y|+\sqrt{|z|}) .
$$

Proof. We first get an upper bound. Every group element $g \in \mathcal{H}_{3}$ can be represented as $s^{n} p^{m} q^{l}$ corresponding to the triple $(x, y, z)=(n, m, n m+l)$. It can be verified that $s^{k} p^{k} s^{-k} p^{-k}=q^{k^{2}}$. Therefore, the length of $q^{l}$ is at most $6 \sqrt{|l|} \leqslant 6 \sqrt{|z|}+3|n|+3|m|$. For $C_{2}=6$ we obtain the required upper bound. Let us prove now a lower bound. If $d_{A}(g)=r$ for an element $g$ corresponding to a triple $(x, y, z)$, then $|x|,|y| \leqslant r$ and $|z| \leqslant r+r^{2}$. For $C_{1}=\frac{1}{4}$ we obtain the required lower bound.

Let us construct a Cayley automatic representation of the Heisenberg group $\mathcal{H}_{3}$ which is compatible with Definition [3. For a given $g \in \mathcal{H}_{3}$ corresponding to a triple $(x, y, z)$ we construct the word $w=u v$ which is the concatenation of two words $u, v \in S^{*}$ constructed as follows. We put $u=p^{y}$. Let $b_{x}$ and $b_{z}$ be the binary representations of the integers $|x|$ and $|z|$ (with the least significant digits first). We put $b$ to be $b_{x} \otimes b_{z}$ with the padding symbol $\diamond$ changed to 0 . The word $b$ is a word over the alphabet consisting of the symbols $\left(\begin{array}{l}0 \\ 0\end{array}\right),\left(\begin{array}{l}0 \\ 1\end{array}\right),\left(\begin{array}{l}1 \\ 0\end{array}\right),\left(\begin{array}{l}1 \\ 1\end{array}\right)$.

Replacing the symbols $\left(\begin{array}{l}0 \\ 0\end{array}\right),\left(\begin{array}{l}0 \\ 1\end{array}\right),\left(\begin{array}{l}1 \\ 0\end{array}\right),\left(\begin{array}{l}1 \\ 1\end{array}\right)$ in $b$ by the words $e e, e q$, se and $s q$ we obtain a word $b^{\prime} \in\{e, s, q\}^{*}$. If $x \geqslant 0$ and $z \geqslant 0$, then we put $v=b^{\prime}$. If $x<0$ or $z<0$, then $v$ is obtained from $b^{\prime}$ by replacing the symbols $s$ and $q$ to the symbols $s^{-1}$ and $q^{-1}$, respectively. For example, the triple $(3,-3,-4)$ is represented by the word $p^{-1} p^{-1} p^{-1}$ seseeq $^{-1}$. The set of all such words $w$ is a regular language $L \subseteq S^{*}$. Thus, we have constructed the bijection $\psi: L \rightarrow \mathcal{H}_{3}$. It can be verified that $\psi$ provides a Cayley automatic representation of the Heisenberg group $\mathcal{H}_{3}$.

It is worth noting that if $g \in \mathcal{H}_{3}$ corresponds to a triple $(0, y, 0)$, then for the word $w=\psi^{-1}(g)$ we have $\psi(w)=\pi(w)$. That is, the maps $\pi$ and $\psi$ coincide if restricted on the cyclic subgroup $\langle p\rangle \leqslant \mathcal{H}_{3}$.

Theorem 17. The Heisenberg group $\mathcal{H}_{3} \in \mathcal{B}_{\mathfrak{e}}$. Moreover, for any $f \prec \sqrt[3]{n}$, $\mathcal{H}_{3} \notin \mathcal{B}_{f}$.

Proof. Let $h$ be the function given by (11) with respect to the Cayley automatic representation $\psi: L \rightarrow \mathcal{H}_{3}$ constructed above. We will show that $h \asymp \mathfrak{e}$. Although 
for the first statement of the theorem it is enough to show that $h \preceq \mathfrak{e}$, the inequality $\mathfrak{e} \preceq h$ guarantees that we cannot get a better result using just the representation $\psi$. Let $w=u v \in L^{\leqslant n}$ and $g=\psi(w)$ be the group element of $\mathcal{H}_{3}$ corresponding to a triple $(x, y, z)$. By Proposition 16, there exists a constant $C_{2}$ such that $d_{A}(g) \leqslant C_{2}(|x|+|y|+\sqrt{|z|}) \leqslant C_{2}\left(2^{|v|}+|u|+\sqrt{2^{|v|}}\right) \leqslant 2 C_{2} 2^{|u|+|v|} \leqslant$ $2 C_{2} \exp (|w|) \leqslant 2 C_{2} \exp (n)$. Therefore, $h \preceq \mathfrak{e}$ which implies that $\mathcal{H}_{3} \in \mathcal{B}_{\mathfrak{e}}$.

Let us show now that $\mathfrak{e} \preceq h$. Let $g_{i}=s^{i}, i \geqslant 2$. The length of the corresponding word $w_{i}=\psi^{-1}\left(g_{i}\right)$ is equal to the doubled length of the binary representation of $i$. We have $d_{A}\left(\pi\left(w_{i}\right), \psi\left(w_{i}\right)\right)=d_{A}\left(\pi\left(w_{i}\right)^{-1} s^{i}\right)=d_{A}\left(s^{n_{i}}\right)$ for some positive integer $n_{i}$. Clearly, there exists a constant $C$ such that $n_{i} \geqslant C i$. The group element $s^{n_{i}}$ corresponds to the triple $\left(n_{i}, 0,0\right)$. By Proposition [16 we have $d_{A}\left(s^{n_{i}}\right) \geqslant C_{1} n_{i}$. Therefore, there exists a constant $C^{\prime}>0$ such that $d_{A}\left(s^{n_{i}}\right) \geqslant C^{\prime} 2^{\frac{\left|w_{i}\right|}{2}}$ for all $i \geqslant 2$. This implies that $\mathfrak{e} \preceq h$. Therefore, $h \asymp \mathfrak{e}$.

Let us show now the second statement of the theorem. Repeating exactly the same argument as used in Theorem 13, we conclude that there exists a function $\ell(n) \prec \sqrt[3]{n}$ for which the inequality $D(n) \preceq n^{2} D(\ell(n))$ holds, where $D(n)$ is the Dehn function of $\mathcal{H}_{3}$. For the group $\mathcal{H}_{3}$ the Dehn function is at most cubic; specifically for the presentation $\mathcal{H}_{3}=\langle s, p, q| s^{-1} p^{-1} s p=q, s q=q s, p q=$ $q p\rangle, D(n) \leqslant n^{3}$ (see [10, $\left.\S 8.1\right]$ ). Therefore, $D(n) \preceq n^{2} \ell(n)^{3}$. Let us show that $n^{2} \ell(n)^{3} \prec n^{3}$. It can be seen that $n^{2} \ell(n)^{3} \preceq n^{3}$. Assume that $n^{3} \preceq n^{2} \ell(n)^{3}$. Then, for all sufficiently large $n$ and some constants $K$ and $M$ we have: $n^{3} \leqslant$ $K n^{2} \ell(M n)^{3}$. This implies that $\sqrt[3]{n} \leqslant \sqrt[3]{K} \ell(M n)$. Therefore, $\sqrt[3]{n} \preceq \ell(n)$ which contradicts to the inequality $\ell(n) \prec \sqrt[3]{n}$. Thus, $D(n) \preceq n^{2} \ell(n)^{3} \prec n^{3}$ which implies that $D(n) \prec n^{3}$. The last inequality contradicts to the fact that the Dehn function is at least cubic (see [10, § 8.1]) which implies that $n^{3} \preceq D(n)$.

\section{Discussion}

In this paper we proposed a way to measure closeness of Cayley automatic groups to the class of automatic groups. We did this by introducing the classes of Cayley automatic groups $\mathcal{B}_{f}$ for the functions $f \in \mathfrak{F}$. In Theorem 9 we characterized non-automatic groups by showing that for any such group $G$ in some class $\mathcal{B}_{f}$ the function $f$ must be unbounded. We studied then the cases of the BaumslagSolitar groups $B S(p, q), 1 \leqslant p<q$, the lamplighter group and the Heisenberg group $\mathcal{H}_{3}$. In Theorems 13 and 15 we proved that the Baumslag-Solitar groups and the lamplighter group are in the class $\mathcal{B}_{\mathfrak{i}}$ and they cannot belong to any class $\mathcal{B}_{f}$ for which $f \prec \mathfrak{i}$. For the Heisenberg group $\mathcal{H}_{3}$ in Theorem 17 we proved that $\mathcal{H}_{3} \in \mathcal{B}_{\mathfrak{e}}$, but we could only prove that it cannot belong to any class $\mathcal{B}_{f}$ for which $f \prec \sqrt[3]{n}$. The following questions are apparent from the results obtained in this paper.

- Is there any unbounded function $f \prec \mathfrak{i}$ for which the class $\mathcal{B}_{f}$ contains a non-automatic group?

- Is there any function $f \prec \mathfrak{e}$ for which $\mathcal{H}_{3} \in \mathcal{B}_{f}$ ?

- Is there any characterization of a class $\mathcal{B}_{f}$, where $f$ is an unbounded function? 


\section{Acknowledgments}

The authors thank the referees for useful comments.

\section{References}

1. Baumslag, G., Solitar, D.: Some two-generator one-relator non-Hopfian groups. Bulletin of American Mathematical Society 68, 199-201 (1962)

2. Berdinsky, D., Khoussainov, B.: On automatic transitive graphs. In: Shur, A., Volkov, M. (eds.) Developments in Language Theory 2014, Lecture Notes in Computer Science, vol. 8633, pp. 1-12. Springer International Publishing (2014)

3. Berdinsky, D., Khoussainov, B.: Cayley automatic representations of wreath products. International Journal of Foundations of Computer Sceince 27(2), 147-159 (2016)

4. Bérubé, S., Palnitkar, T., Taback, J.: Higher rank lamplighter groups are graph automatic. Journal of Algebra 496, 315-343 (2018)

5. Blachere, S.: Word distance on the discrete Heisenberg group. Colloquium Mathematicum 95(1), 21-36 (2003)

6. Blumensath, A.: Automatic Structures. Diploma Thesis, RWTH (1999)

7. Blumensath, A., Grädel, E.: Automatic structures. In 15th Symposium on Logic in Computer Science LICS pp. 51-62 (2000)

8. Burillo, J., Elder, M.: Metric properties of Baumslag-Solitar groups. International Journal of Algebra and Computation 25(5), 799-811 (2015)

9. Cleary, S., Taback, J.: Dead end words in lamplighter groups and other wreath products. The Quarterly Journal of Mathematics 56(2), 165-178 (Jun 2005)

10. Epstein, D.B.A., Cannon, J.W., Holt, D.F., Levy, S.V.F., Paterson, M.S., Thurston, W.P.: Word Processing in Groups. Jones and Barlett Publishers. Boston, MA (1992)

11. Kargapolov, M.I., Merzljakov, J.I.: Fundamentals of the theory of groups. SpringerVerlag Berlin Heidelberg New York (1979)

12. Kharlampovich, O., Khoussainov, B., Miasnikov, A.: From automatic structures to automatic groups. Groups, Geometry, and Dynamics 8(1), 157-198 (2014)

13. Khoussainov, B., Minnes, M.: Three lectures on automatic structures. Proceedings of Logic Colloquium pp. 132-176 (2007)

14. Khoussainov, B., Nerode, A.: Automatic presentations of structures. In: Leivant, D. (ed.) Logic and Computational Complexity, Lecture Notes in Computer Science, vol. 960, pp. 367-392. Springer Berlin Heidelberg (1995)

15. Khoussainov, B., Nerode, A.: Open questions in the theory of automatic structures. Bulletin of the EATCS 94, 181-204 (2008)

16. Khoussainov, B., Nies, A., Rubin, S., Stephan, F.: Automatic structures: richness and limitations. Logical Method in Computer Science 3(2:2), 1-18 (2007)

17. Lyndon, R.C., Schupp, P.E.: Combinatorial group theory. Springer-Verlag Berlin Heidelberg New York (1977)

18. Nies, A., Thomas, R.M.: FA-presentable groups and rings. Journal of Algebra 320, 569-585 (2008)

19. Oliver, G.P., Thomas, R.M.: Automatic presentations for finitely generated groups. In: Diekert, V., Durand, B. (eds.) STACS 2005, Lecture Notes in Computer Science, vol. 3404, pp. 693-704. Springer Berlin Heidelberg (2005) 
20. Pelecq, L.: Isomorphismes et automorphismes des graphes context-free, équationnels et automatiques. PhD Thesis, Bordeaux 1 University (1997)

21. Roe, J.: Lectures on Coarse Geometry, University Lecture Series, vol. 31. American Mathematical Society (2003)

22. Rubin, S.: Automatic Structures. PhD Thesis, The University of Auckland (2004)

23. Sénizergues, G.: Definability in weak monadic second-order logic of some infinite graphs. Automata Theory: Infinite Computations, vol. 9202, p. 16. Dagstuhl Seminar, Wadern, Germany (1992)

24. Vershik, A.: Numerical characteristics of groups and corresponding relations. Journal of Mathematical Sciences 107(5), 4147-4156 (2001) 